\title{
Clinical and operational value of the extensively drug-resistant tuberculosis definition
}

\author{
G.B. Migliori*, G. Besozzi ${ }^{\#}$, E. Girardi ${ }^{\uparrow}$, K. Kliiman ${ }^{+}$, C. Lange ${ }^{\S}$, O.S. Toungoussova ${ }^{f}$, \\ G. Ferrara**, D.M. Cirillo ${ }^{\# \#, ~ A . ~ G o r i ~}{ }^{\top \uparrow}$, A. Matteelli ${ }^{++}$, A. Spanevello ${ }^{\dagger}$, L.R. Codecasa ${ }^{\S \S}$, \\ M.C. Raviglione ${ }^{f f}$ and SMIRA/TBNET Study Group
}

ABSTRACT: Currently, no information is available on the effect of resistance/susceptibility to first-line drugs different from isoniazid and rifampicin in determining the outcome of extensively drug-resistant tuberculosis (XDR-TB) patients, and whether being XDR-TB is a more accurate indicator of poor clinical outcome than being resistant to all first-line anti-tuberculosis (TB) drugs.

To investigate this issue, a large series of multidrug-resistant TB (MDR-TB) and XDR-TB cases diagnosed in Estonia, Germany, Italy and the Russian Federation during the period 1999-2006 were analysed. Drug-susceptibility testing for first- and second-line anti-TB drugs, quality assurance and treatment delivery was performed according to World Health Organization recommendations in all study sites.

Out of 4,583 culture-positive TB cases analysed, 361 (7.9\%) were MDR and 64 (1.4\%) were XDR. XDR-TB cases had a relative risk (RR) of 1.58 to have an unfavourable outcome compared with MDR-TB cases resistant to all first-line drugs (isoniazid, rifampicin ethambutol, streptomycin and, when tested, pyrazinamide), and an RR of 2.61 compared with "other" MDR-TB cases (those susceptible to at least one first-line anti-TB drug among ethambutol, pyrazinamide and streptomycin, regardless of resistance to the second-line drugs not defining XDR-TB).

The emergence of extensively drug-resistant tuberculosis confirms that problems in tuberculosis management are still present in Europe. While waiting for new tools which will facilitate management of extensively drug-resistant tuberculosis, accessibility to quality diagnostic and treatment services should be urgently ensured and adequate public health policies should be rapidly implemented to prevent further development of drug resistance.

KEYWORDS: Clinical value, drug resistance, extensively drug-resistant tuberculosis, multidrugresistant tuberculosis, tuberculosis

E xtensively drug-resistant tuberculosis (XDR-TB) is defined as resistance to at least rifampin $(\mathrm{R})$ and isoniazid $(\mathrm{H}$; this is the definition of multidrug-resistant tuberculosis (MDR-TB)), in addition to any fluoroquinolone, and at least one of the three injectable antituberculosis (TB) drugs (capreomycin, kanamycin and amikacin). The XDR-TB definition was made on the assumption that these classes of drugs are essential to successfully treat a case of TB, although evidence of its clinical relevance was not available at the time [1-4]

In a preliminary analysis of European patients, the current authors recently demonstrated higher

STATEMENT OF INTEREST: None declared. probability of death and worse outcomes in XDR-TB cases when compared with MDR-TB cases [5].

Previous studies demonstrated that among MDRTB cases the probability of achieving treatment success varies, depending on the number of firstline drugs the patient is susceptible to [6]. However, it is not known whether XDR-TB is a more accurate indicator of poor clinical outcome than being resistant to all first-line anti-TB drugs [6]. In fact, there is no information available on the effect of resistance/susceptibility to first-line drugs different from $H R$ in determining the outcome of XDR-TB patients. To investigate this issue a larger series of MDR-TB and XDR-TB cases diagnosed in Western and Eastern European countries was analysed.
AFFILIATIONS

*WHO Collaborating Centre for TB and Lung Diseases, Fondazione $S$

Maugeri, Care and Research Institute, Tradate,

${ }^{\#}$ E. Morelli Hospital, Reference Hospital for MDR and HIV TB, Sondalo,

"National Institute for Infectious Diseases L. Spallanzani, Rome, ${ }^{f}$ Fondazione S. Maugeri, Care and Research Institute, Cassano delle Murge,

${ }^{* \star}$ University of Perugia, Internal Medicine, Section of Respiratory Diseases, Perugia,

\#\#Supranational Reference Laboratory, S. Raffaele Institute, "San Paolo Hospital, University of Milan, and

${ }^{5 \S}$ TB Reference Centre, Villa Marelli Institute, Niguarda Hospital, Milan, and ${ }^{++}$University of Brescia, Brescia, Italy. +University of Tartu, Tartu, Estonia. ${ }^{\S}$ Division of Clinical Infectious Diseases, Medical Clinic, Research Center Borstel, Borstel, Germany. ${ }^{f f}$ Stop TB Dept, World Health Organization, Geneva, Switzerland.

\section{CORRESPONDENCE}

G.B. Migliori, WHO Collaborating Centre for TB and Lung Diseases, Fondazione S. Maugeri, Care and Research Institute, via Roncaccio 16, 21049 Tradate, Italy. Fax: 39 331829402. E-mail: gbmigliori@ fsm.it

Received: June 252007

Accepted after revision: July 232007

SUPPORT STATEMENT

The study was funded in part by a grant from the Superior Institute of Health, Centre for Disease Control, Ministry of Health, Rome, Italy.

European Respiratory Journal Print ISSN 0903-1936 Online ISSN 1399-3003 


\section{METHODS}

Data from all culture confirmed TB cases diagnosed consecutively by the TB clinical reference centres in Estonia (Tallin and Tartu), Germany (Borstel, Grosshansdorf and BadLippspringe), Italy (Sondalo, Milan and Rome) and a NorthEastern region of the Russian Federation (Archangels Oblast) were analysed.

Clinical outcomes (available on the original clinical records) were measured as part of an ad hoc study performed in the previously mentioned countries during the period 1999-2006 (Italy and Germany: 2003-2006; Estonia: 2001-2004; Archangels Oblast: 1999-2001) [5, 7, 8]. Drug-susceptibility testing (DST) for first- and second-line anti-TB drugs was performed according to World Health Organization (WHO) recommendations by quality-assured laboratories and was re-tested at the WHO's Supranational Reference Laboratories (Rome/Milan, Borstel, Stockolm and Oslo) $[9,10]$. In Italy, Germany and Estonia the BACTEC $^{\mathrm{TM}}$ MGIT $960^{\mathrm{TM}}$ TB System (Becton Dickinson Diagnostic Systems, Sparks, MD, USA) was used to test firstline drugs and the proportion method on Lowenstein-Jensen was used to test second-line drugs in all centres. In Archangels Oblast the proportion method on Lowestein-Jensen medium was used. In Oslo, DST for both first- and second-line drugs was carried out using the BACTEC 460 TB System (Becton Dickinson Diagnostic Systems).

In all countries, regimens to treat MDR-TB and XDR-TB cases were tailored to the DST results according to WHO recommendations, the main categories of second-line drugs being generally available to treat patients during the study period (injectable agents: amikacin, capreomycin and kanamycin; fluoroquinolones; second-line oral agents: ethionamide/ prothionamide; para-aminosalycilic acid and cycloserine). Third-line agents (e.g. amoxicillin/clavulanicacid, clarithromycin, clofazimine) were not available in Archangels Oblast.

MDR-TB cases resistant to all first-line drugs were defined as those resistant to $H, R$, ethambutol, streptomycin and, when tested, pyrazinamide. Other MDR-TB cases were those susceptible to at least one first-line anti-TB drug among ethambutol, pyrazinamide and streptomycin (regardless of resistance to the second-line drugs not defining XDR-TB).

Outcomes were compared using the Chi-squared test (categorical variables) in cases achieving a final outcome (different from default, transferred out and still on treatment), and using the Kaplan-Meier curve where appropriate.

\section{RESULTS}

Out of 4,583 culture-positive TB cases analysed (Italy: 2,140; Germany: 748; Estonia: 900; Archangels Oblast: 795), 361 (7.9\%) were MDR (Italy: 83; Germany: 43; Estonia: 194; Archangels Oblast: 41 ) and 64 (1.4\%) were XDR (Italy: 8; Germany: 3; Estonia: 53; Archangels Oblast: 0$)$. In Italy, $1.46 \%$ of all the notified culture-positive cases were MDR (4.2\% in the present study); in Germany, 2.1\% of culture-positive cases were MDR (6.1\% in the present study); in Estonia they were $27.4 \%$ and in Archangels Oblast $5.2 \%$, as all cases were included in the study. In total, 178 (49.3\%) out of 361 MDR-TB cases and 48 (75\%) out of 64 XDR-TB cases were re-treatment cases $(p<0.001)$. Out of 341 MDR-TB cases tested for HIV , 17 (5\%) were HIV infected, as were two (3.2\%) out of the 61 XDR-TB cases. Out of 361 MDRTB cases, $267(74 \%)$ were resistant to all first-line drugs, 51 (14.1\%) were resistant to $\mathrm{H}, \mathrm{R}$ and streptomycin, 19 (5.3\%) to $\mathrm{H}$, $\mathrm{R}$ and ethambutol, and $24(6.6 \%)$ to HR.

Out of 64 XDR-TB cases, none were resistant to HR only, one case was resistant to fluoroquinolone and one was resistant to injectable drugs. A total of $58(90.6 \%)$ were resistant to all firstline drugs, (plus, eventually, other second-line drugs) and 6 $(9.4 \%)$ were resistant to HR plus ethambutol or streptomicin and/or other second-line drugs.

Included in the outcome analysis were 240 MDR-TB cases (187 were resistant to all first-line drugs) and 48 XDR-TB cases achieving a final outcome.

XDR-TB cases were more likely to be resistant to all first-line drugs than MDR-TB cases $(\mathrm{p}<0.005)$. The cases excluded from the analysis were equally distributed among groups. Patients still receiving treatment were as follows. XDR-TB: eight out of 64; MDR-TB resistant to all first-line drugs: 38 out of 267; other MDR: 31 out of 94. Patients who defaulted/transferred out were as follows. XDR-TB: eight out of 64; MDR-TB resistant to all first-line drugs: 42 out of 267; other MDR: 10 out of 64 .

No difference in the profile of drug resistance for second-line drugs was found among the groups analysed, excluding the XDR-defining second-line drugs (XDR-TB: mean 1.3, median 1; MDR-TB resistant to all first-line drugs: mean 1.3, median 1; other MDR-TB: mean 0.8, median 1). Details on outcomes by resistance pattern are summarised in table 1 .

At the univariate analysis, XDR-TB cases had significantly worse outcomes than MDR-TB cases resistant to all first-line drugs and other MDR-TB cases.

XDR-TB cases had a relative risk (RR) of 1.58 to have an unfavourable outcome compared with MDR-TB cases resistant to all first-line drugs (95\% confidence interval (CI) 1.14-2.20, 26 out of 48 versus 64 out of 187; p <0.05) and an RR of $2.61(95 \%$ CI $1.45-4.69,26$ out of 48 versus 11 out of 53 ; $\mathrm{p}<0.001$ ) compared with other MDR-TB cases.

MDR-TB cases resistant to all first-line drugs were more likely to have an unfavourable outcome than other MDR-TB cases (death or failure: 64 out of 187 versus 11 out of 53, RR 1.65, 95\% CI 0.94-2.89), although the difference was not significant at the conventional p 0.05 level $(p=0.06)$. The difference was statistically significant if patients still on treatment were not removed from the analysis (64 out of 225 versus 11 out of 84; $p<0.01)$. This analysis is performed under the assumption that the patients still on regular treatment will achieve a successful treatment outcome. If the opposite is assumed, i.e. that all patients still on treatment achieve an unsuccessful treatment outcome, no greater statistically significant difference is detected between the two groups. The difference in treatment outcomes among the three groups also remains significant after adjusting for age and country of diagnosis.

Using the Kaplan-Meier analysis, the time to treatment success is significantly different among the three groups, with the lowest rate of treatment success in the $\mathrm{XDR}-\mathrm{TB}$ group $(\mathrm{p}<0.005$; fig 1$)$. 


\begin{tabular}{|c|c|c|c|c|c|c|}
\hline $\begin{array}{l}\text { Outcom } \\
\text { Estonia, }\end{array}$ & $\begin{array}{l}\text { es of extensively } \\
\text { Germany, Italy ar }\end{array}$ & $\begin{array}{l}\text { Sistant t } \\
\text { Russiar }\end{array}$ & $\begin{array}{l}\text { sis (XDF } \\
\text { ion }\end{array}$ & nultidru & ant tubercul & (MDR-TB) cases in \\
\hline & Treatment success & Died & Default & Failure & Transferred & $\begin{array}{c}\text { Total patients } \\
\text { completing treatment }\end{array}$ \\
\hline XDR-TB $\#$ & $22(39.3)$ & $14(25.0)$ & $8(14.3)$ & $12(21.4)$ & $0(0.0)$ & 56 \\
\hline $\begin{array}{l}\text { MDR-TB resistant to all } \\
\text { first-line drugs }\end{array}$ & $123(53.7)$ & $35(15.3)$ & $39(17.0)$ & $29(12.7)$ & $3(1.3)$ & 229 \\
\hline Other MDR-TB ${ }^{+}$ & $42(66.7)$ & $8(12.7)$ & $10(15.9)$ & $3(4.8)$ & $0(0.0)$ & 63 \\
\hline Total & $187(53.7)$ & $57(16.4)$ & 57 (16.4) & $44(12.6)$ & $3(0.9)$ & 348 \\
\hline
\end{tabular}

Data are presented as $\mathrm{n}(\%)$ or $\mathrm{n} .{ }^{*}$ : resistance to at least rifampin and isoniazid (definition of MDR-TB) in addition to any fluoroquinolone, and at least one of the three injectable anti-TB drugs (capreomycin, kanamycin and amikacin); ' : cases resistant to isoniazid, rifampicin ethambutol, streptomycin and, when tested, pyrazinamide: : cases susceptible to at least one first-line anti-TB drug. Default and transferred cases included in this table were removed from the analysis presented in the text.

\section{DISCUSSION}

This is the first study to show in a large cohort from four European countries, at low HIV prevalence, that XDR-TB cases have a clinical outcome worse than MDR-TB cases resistant to all first-line anti-TB drugs, and that susceptibility to one or more first-line drugs increases the probability to treat successfully MDR-TB cases. The results also demonstrate the possible existence of a "continuum" of severity in terms of clinical outcome among XDR-TB, MDR-TB resistant to all first-line drugs and other MDR-TB cases.

The results of the current study, which are consistent with those of a recently performed laboratory-based survey [8], show the following. 1) XDR-TB cases with a resistance pattern strictly corresponding to the definition (e.g. H, R, one fluoroquinolone and one injectable drug) are not frequently identified in the clinical practice, as second-line drugs are introduced when drug resistance to all first-line drugs is likely

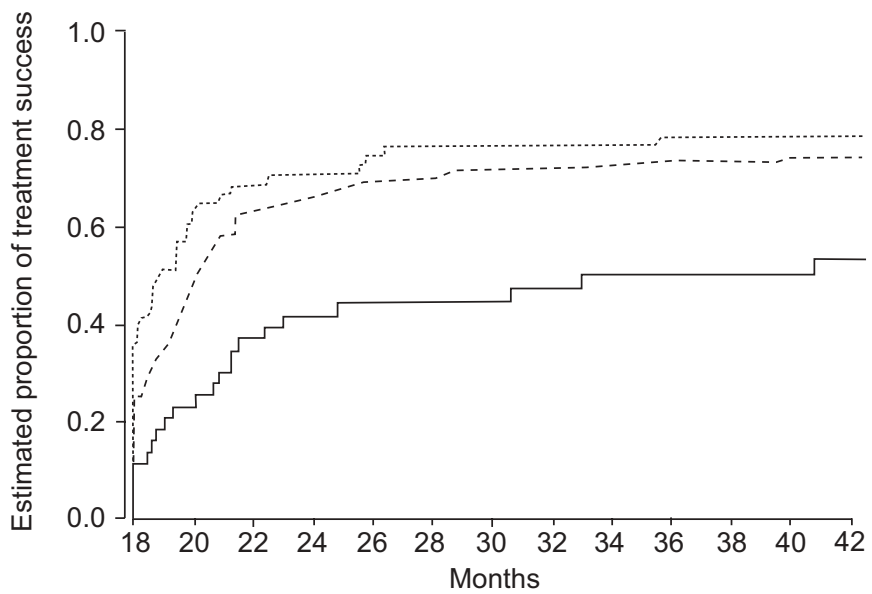

FIGURE 1. A Kaplan-Meier plot showing estimated proportion of treatment success (cure plus treatment completion) according to the drug-resistance profile in Estonia, Germany, Italy and the Russian Federation. ........: other mutidrugresistant tuberculosis (MDR-TB) cases; - - - - -: MDR-TB cases resistant to all firstline drugs; _ _ extensively drug-resistant tuberculosis cases. to have occurred. 2) The occurrence of XDR-TB, as currently defined, has both a clinical value (predicting poor outcome) and an operational significance (confirming the loss of first-line drugs coupled with key second-line drugs).

Limitations of the study include the following. First, the observation that data are representative in only two of the settings surveyed (Estonia and Archangels Oblast). In Italy and Germany the prevalence of MDR-TB in TB clinical reference centres is higher than the prevalence detected at national level.

Secondly, since $16.4 \%$ of patients were lost to follow-up, their outcome is not well characterised. Thirdly, the difference in outcomes between MDR-TB resistant to all first-line drugs versus other MDR-TB cases reached only borderline significance under the assumption that patients still receiving regular treatment will reach a successful outcome. In the current authors' opinion this assumption is more likely to represent the truth than the opposite, i.e. that all patients still on treatment will have an unsuccessful outcome. Due to the difficulty in raising large numbers on a relatively uncommon form of disease, such as MDR-TB/XDR-TB, global studies will be necessary to give a final answer to this question.

Finally, although DST for second-line drugs in the present study were quality controlled by WHO Supranational Reference Laboratories, some caution is always needed when interpreting results in relation to XDR-TB. Although protocols to standardise DST for second-line drugs are presently being developed, universally accepted proficiency testing does not exist.

The fact that the results from Italy and Germany [5] remain consistent after including data from Eastern European countries suggests that the study results are robust. The negative impact of TB treatment mismanagement (and suboptimal infection control in congregate settings) $[5,11]$ in selecting resistant mutants in Europe is further confirmed by the observation that 75 and $49.3 \%$ of XDR-TB and MDR-TB cases, respectively, were previously treated for TB.

Further information on extensively drug-resistant tuberculosis will hopefully be available in the next few years when surveillance systems will be equipped to identify all the existing extensively drug-resistant tuberculosis cases and to 
monitor their risk factors and outcomes [11]. At the same time, the emergence of extensively drug-resistant tuberculosis confirms that problems in tuberculosis management are still present in Europe. While waiting for new tools that will facilitate the management of extensively drug-resistant tuberculosis, accessibility to quality diagnostic and treatment services should be urgently ensured and adequate public health policies should be rapidly implemented to prevent further development of drug resistance.

\section{ACKNOWLEDGEMENTS}

Members of the Multicentre Italian Study on Resistance to Antituberculosis drugs (SMIRA)/ TuBerculosis Network in Europe Trialsgroup (TBNET) are: J. Ortmann (Bad Lippspringe Hospital, Bad Lippspringe, Germany), D. Kirsten (Großhansdorf Hospital, Großhansdorf, Germany), S. Ruesch-Gerdes (Supranational Reference Laboratory, Borstel, Germany), F. Piana (S. Raffaele Hospital, Milan, Italy), M. Ferrarese (Villa Marelli Institute, Milan, Italy), G. De Iaco (Sondalo Hospital, Sondalo, Italy), S. De Lorenzo (Sondalo Hospital), P. Troupioti (Sondalo Hospital), L. Fattorini (Supranational Reference Laboratory/Superior Institute of Health, Rome, Italy), E. Iona (Supranational Reference Laboratory/Superior Institute of Health), A. Gualano (INMI L. Spallanzani, Rome, Italy), P. De Mori (INMI L. Spallanzani), R. Centis (WHO Collaborating Centre for TB and Lung Disease, Fondazione S. Maugeri, Care and Research Institute, Tradate, Italy), M. Danilovits (University of Tartu, Tartu, Estonia), V. Hollo (University of Tartu) and A. Mariandyshev (Northern State Medical University, Archangels, Russia).

\section{REFERENCES}

1 Extensively drug-resistant tuberculosis (XDR-TB): recommendations for prevention and control. Weekly Epidemiol Rec 2006; 81: 430-432.

2 Centers for Disease Control and Prevention (CDC). Emergence of Mycobacterium tuberculosis with extensive resistance to second-line drugs - worldwide, 2002-2004. MMWR Morb Mortal Wkly Rep 2006; 55: 301-305.
3 Gandhi NR, Moll A, Sturm AW, et al. Extensively drugresistant tuberculosis as a cause of death in patients coinfected with tuberculosis and HIV in a rural area of South Africa. Lancet 2006; 368: 1575-1580.

4 Migliori GB, Loddenkemper R, Blasi F, Raviglione MC. 125 years after Robert Koch's discovery of the tubercle bacillus: the new XDR-TB threat. Is "science" enough to tackle the epidemic? Eur Respir J 2007; 29: 423-427.

5 Migliori GB, Ortmann J, Girardi E, et al. Extensively drugresistant tuberculosis, Italy and Germany. Emerging Infectious Diseases. Www.cdc.gov/EID/content/13/5/ 780.htm. Date last updated: June 25, 2007. Date last accessed: June 25, 2007.

6 Espinal MA, Kim SJ, Suarez PG, et al. Standard short-course chemotherapy for drug-resistant tuberculosis. Treatment outcomes in 6 countries. JAMA 2000; 283: 2537-2545.

7 Kliiman K, Centis, R, Migliori GB, et al. Evaluation of DOTS-Plus in Estonia. Int J Tuberc Lung Dis 2005; 9: Suppl. $1, \mathrm{~S} 133$.

8 Toungoussova OS, Nizovtseva NI, Mariandyshev AO, Caugant DA, Sandven P, Bjune G. Impact of drug-resistant Mycobacterium tuberculosis on treatment outcome of culture-positive cases of tuberculosis in the Archangel Oblast, Russia, in 1999. Eur J Clin Microbiol Infect Dis 2004; 23: 174-179.

9 Laszlo A, Rahman M, Espinal M, Raviglione M, the WHO/ IUATLD Network of Supranational Reference Laboratories. Quality assurance programme for drug susceptibility testing of Mycobacterium tuberculosis in the WHO/IUATLD Supranational Reference Laboratory Network: five rounds of proficiency testing, 1994-1998. Int J Tuberc Lung Dis 2002; 6: 748-756.

10 Shah NS, Wright A, Bai G-H, et al. Worldwide emergence of extensively drug-resistant tuberculosis. Emerg Infect Dis 2007; 13: 380-387.

11 Centers for Disease Control and Prevention (CDC). Extensively drug-resistant tuberculosis. United States, 1993-2006. MMWR Morb Mortal Wkly Rep 2007; 56: 250-253. 